\title{
The quest for quantitative microscopy
}

\author{
With the aid of informatics, microscopy is in the midst of a crucial evolution into a more \\ quantitative and powerful technique.
}

Microscopy has historically been a qualitative technique, but the transition to digital microscopy and advances in camera technology, coupled with new labeling and imaging methods, are making it easier to extract meaningful quantitative data from images. Computational techniques are central to this process. The transition of microscopy into a more quantitative technique will bring important scientific benefits in the form of new applications and improved performance and reproducibility.

Current limitations in bioimage-informatics techniques are preventing sophisticated optical methods from realizing their full potential. For example, the algorithms necessary to localize individual fluorophores in super-resolution microscopy data are still in their infancy, and the lack of tools to automatically reconstruct neuronal networks from $3 \mathrm{D}$ image stacks is hindering progress in neuroscience.

More researchers are developing computational solutions to bioimaging challenges; these projects are increasingly part of collaborative open-source community efforts. To promote dialogue between computational tool developers and microscopy users, and to broaden awareness of the computational challenges in bioimaging and the needs of the informatics community, this issue contains a special focus on bioimage informatics. In an introductory Commentary (p659), a central figure in early genome bioinformatics, Gene Meyers, remarks on how the current climate in bioimage informatics resembles the early days of that field.

In spite of the prevalence of microscopy and image analysis in biology, persuading the larger research community to recognize bioimage informatics as a distinct scientific discipline entails profound challenges (p661). The kind of effort required to create a successful bioimage-informatics tool is different from that needed for success in conventional biological research.

The primary users of bioimage-informatics tools are biologists with little or no programming or informatics training and who are operating their own microscopes and analyzing their own data. They require usable, wellengineered and well-supported tools that are flexible enough to easily adapt to their particular needs (p666). But developing and providing ongoing support for userfriendly tools, although essential for bioimaging, presents challenges to funders and institutions.

Many in the community recognize the importance of developing these tools. This is highlighted in this issue in articles describing algorithm solutions to imaging challenges or presenting generalist image-analysis platforms that can be used to distribute such algorithm solutions to biologists in user-friendly packages.

Encouragingly, some institutions are devoting substantial resources in support of major open-source software tools. Funders are also making efforts: the US National Institutes of Health runs a 'Continued Development and Maintenance of Software' program, and the US National Science Foundation recently announced the 'Software Infrastructure for Sustained Innovation' program.

With sufficient support for bioimage informatics, we expect that the days of manually chosen 'representative' images are numbered. Not only will such images be replaced by quantitative measures based on the underlying image data, but even the example images shown in research articles will be either computed representations or computationally chosen representative images. As a result, the level of trust placed in imaging results should increase.

In such a scenario, however, statistical rigor and clear reporting are critical. In their absence, quantification has limited value, and there is the risk that the mere act of such quantification could lead to false confidence in the results. It is crucial that uncertainties be communicated alongside absolute numbers or computationally generated representations. The community should start discussing what efforts are needed to standardize and statistically assess image data. There is a strong foundation of statistical methods for testing and reporting the significance of simple numerical data, and if these methods cannot be translated to more complex image data-perhaps something comparable to a $P$ value for a representative imagealternatives must be implemented or developed.

The statistical rigor required in drug discovery or clinical studies can often be used to inform data analysis and reporting practices in basic research, but even though pharmaceutical companies use high-throughput imaging data, they typically transform the image data into simple numerical values representative of the outcome of each assay condition. In contrast, imaging experiments intended for biological insight must often retain complex information. Now is the time to start tackling these challenges, and doing so requires a strong community of bioimage informaticians and biologists working together.

Microscopy is in the midst of a period of remarkable technological development as researchers-genomes in hand-strive to understand the interplay between a genome and the living physical organism with all its spatial and temporal complexities. Bioimage informatics will play an increasingly important role in bringing the necessary quantitative rigor to these studies. 